\title{
APPROXIMATION OF FUNCTIONS BY GENUINE BERNSTEIN-DURRMEYER TYPE OPERATORS
}

\author{
Tuncer Acar, Ana Maria ACu And Nesibe Manav
}

\begin{abstract}
Very recently, in [4] Chen et. al introduced and considered a new generalization of Bernstein polynomials depending on a patameter $\alpha$. As classical Bernstein operators, these operators also provide interpolation at the end points of $[0,1]$ and they have the linear precision property which means those reproduce the linear functions. In this paper we introduce genuine $\alpha$-Bernstein-Durrmeyer operators. Some approximation results, which include local approximation, error estimation in terms of Ditzian-Totik modulus of smoothness are obtained. Also, the convergence of these operators to certain functions is shown by illustrative graphics using MAPLE algorithms.
\end{abstract}

Mathematics subject classification (2010): 41A10, 41A25, 41A36.

Keywords and phrases: Genuine Bernstein-Durrmeyer operators, rate of convergence, linear positive operators.

\section{REFERENCES}

[1] A. M. ACU, I. RASA, New estimates for the differences of positive linear operators, Numerical Algorithms 73 (3), 775-789, 2016.

[2] S. N. Bernstein, Démonstration du théorème de Weierstrass fondée sur le calcul des probabilités, Communications de la Société Mathematique de Kharkov 13, 1913, 1-2.

[3] L. Beutel, H. Gonska, D. Kacsó, G. TAchev, Variation-diminishing splines revised, in Proc. Int. Sympos. on Numerical Analysis and Approximation Theory (Radu Trâmbiţaş, ed.), Presa Universitară Clujeană, Cluj-Napoca, 2002, 54-75.

[4] X. Chen, J. TAn, Z. Liua, J. Xie, Approximation of functions by a new family of generalized Bernstein operators, J. Math. Anal. Appl. 450 (2017), 244-261.

[5] Z. Ditzian, V. Totik, Moduli of Smoothness, Springer, New York, (1987).

[6] H. GONSKA, R. PĂLTĂNEA, Simultaneous approximation by a class of Bernstein-Durrmeyer operators preserving linear functions, Czechoslovak Math. J. 60 (135), 2010, 783-799.

[7] H. GONSKA, R. PĂLTĂNEA, Quantitative convergence theorems for a class of Bernstein-Durrmeyer operators preserving linear functions, Ukrainian Math. J. 62, 2010, 913-922.

[8] T. N. T. Goodman, A. Sharma, A modified Bernstein-Schoenberg operator, Proc. of the Conference on Constructive Theory of Functions, Varna 1987 (ed. by Bl. Sendov et al.), Sofia: Publ. House Bulg. Acad. of Sci., 1988, 166-173.

[9] V. Gupta AND G. TACHEV, Approximation with Positive Linear Operators and Linear Combinations, Springer 2017.

[10] V. Gupta And R. P. Agarwal, Convergence Estimates in Approximation Theory, Springer 2014.

[11] Z. FInTA, Remark on Voronovskaja theorem for q-Bernstein operators, Stud. Univ. Babeş-Bolyai Math. 56 (2011), 335-339.

[12] A. LuPAŞ, Die Folge der Betaoperatoren, Dissertation, Universität Stuttgart, 1972.

[13] R. PĂLTĂNEA, A class of Durrmeyer type operators preserving linear functions, Ann. Tiberiu Popoviciu Sem. Funct. Equat. Approxim. Convex. (Cluj-Napoca) 5, 2007, 109-117. 